Review Article

\title{
Candidate Urine Peptide Biomarkers for IgA Nephropathy: Where Are We Now?
}

\author{
Karolina Marek-Bukowiec (D), Andrzej Konieczny, Krzysztof Ratajczyk, \\ and Wojciech Witkiewicz
}

Regional Specialist Hospital in Wrocław, Research and Development Center, Wrocław, Poland

Correspondence should be addressed to Karolina Marek-Bukowiec; marek-bukowiec@wssk.wroc.pl

Received 10 September 2017; Revised 29 November 2017; Accepted 12 December 2017; Published 21 January 2018

Academic Editor: Natacha Turck

Copyright (c) 2018 Karolina Marek-Bukowiec et al. This is an open access article distributed under the Creative Commons Attribution License, which permits unrestricted use, distribution, and reproduction in any medium, provided the original work is properly cited.

\begin{abstract}
Early detection, prognosis, and management of IgA nephropathy (IgAN) remain a challenge. Histological examination of renal tissue still comprises the only way to confirm an IgAN diagnosis. It is of great importance to establish noninvasive diagnostic, prognostic, and predictive biomarkers that would improve the clinical care and outcome of patients suffering from IgAN. This review summarises the findings from previous mass spectrometry- (MS-) based studies dedicated to the discovery of urinary peptide profiles specific to IgAN. There is a substantial number of urinary peptides that have been discovered to date, which show promise as biomarkers of IgAN; however, all of them require further, rigorous validation in well-planned studies, involving a large number of subjects who represent diverse and numerous populations.
\end{abstract}

\section{Introduction}

IgA nephropathy (IgAN, Berger's disease) is one of the most predominant variants of primary glomerular disease, ultimately leading to end-stage renal disease (ESRD) in a great proportion of patients (approximately 30-40\%) [1]. Renal biopsy remains the only tool providing a definitive diagnosis of IgAN and the only aid in making optimal therapeutic decisions [2]. However, kidney biopsy carries a considerable risk of potential complications like pain, fever, perirenal hematoma, or hematuria requiring blood transfusion or surgical intervention and is not always feasible due to coexisting conditions (e.g., uncontrolled hypertension, blood coagulation anomalies, anatomic abnormalities, and pregnancy) or the lack of patient consent [3-5]. Thus, there is an urgent need to develop noninvasive biomarkers which are able to provide reliable diagnostic, prognostic, and predictive information in IgAN that would supplement or preferably outperform renal biopsy.

Urinary peptidomics is an emerging and promising field for clinical biomarker discovery in IgA nephropathy. Many previous studies have shown that the urinary peptide profiles of IgAN patients differ significantly both from those suffering from other chronic renal diseases and from other healthy controls [6-8]. Most of the urinary, endogenous peptides stem from the kidneys and the urinary tract and possess a specific expression profile, shaped by physiological and pathophysiological processes ongoing in the urinary system. Thus, a quantitative and qualitative examination of the urinary peptidome of IgA nephropathy patients may facilitate the discovery of specific biomarkers, and in parallel, help to uncover molecular mechanisms driving IgAN. Developing a reliable, clinically useful, urine peptide biomarker/biomarker panel, with the use of mass spectrometry, is not trivial, mostly due to the strong interlaboratory variation in experimental design (e.g., size and composition of cohorts), sample collection (e.g., first versus second morning urine), sample processing (different strategies to isolate urine peptides), and data analysis (various MS platforms and data analysis tools). All of this leads to incomparable datasets and an inability to conduct a meta-analysis to validate the candidate biomarkers [9]. Biomarker discovery in proteomics is also hampered by the complexity of urine samples, a wide range of protein concentration, peptide normalisation difficulties, and 
the confounding effect of numerous variables (e.g., urine $\mathrm{pH}$, age, and diet), influencing the stability and composition of urinary peptide patterns and contributing to false-positive findings [10-12].

In this review, we summarise the current state of the literature regarding urinary peptide profile "specific" for IgA nephropathy, originating from previous mass spectrometrybased studies. We also discuss some of the critical variables that can markedly influence the peptide profile of urinary specimens and confound data interpretation.

\section{Materials and Methods}

In order to summarise the knowledge regarding putative urinary peptide biomarkers of IgA nephropathy identified by mass spectrometry, we have searched the electronic bibliographic databases PubMed and Google Scholar, using advanced search options. The search was performed using various combinations of the following keywords: "IgAN," "IgA nephropathy," "chronic kidney disease," "CKD," "urinary peptidome," "urinary peptides," "urinary proteome," "urinary peptide biomarkers," "urinary peptide pattern," "urinary peptide profile," "proteomic analysis of urine," "urine proteomics," and "proteomic study." We limited our search to original publications written in English, published between 1990 and 2017, including the search terms in the title or in the abstract. The exclusion criteria for the articles were as follows: no full PDF available, review article, letter, comment, case report, or conference abstract. In total, seven original articles were found that met the inclusion criteria.

\section{IgA Nephropathy}

IgA nephropathy is a glomerular disease that can be recognised only by histopathological examination of the renal biopsy specimen, which reveals the presence of dominant or codominant mesangial deposits of $\operatorname{IgA}$ immunoglobulin [13]. The disease can be diagnosed as primary if it is confined only to the kidney or secondary if it comprises a renal manifestation of a systemic disease, like chronic liver disease, diabetes, hypertension, amyloidosis, or lupus [14]. IgAN remains the dominant form of primary glomerulopathies in adults, with a global prevalence of 2.5 cases per 100,000 per year [15]. According to registries of glomerular diseases, IgAN incidence ranges from $5 \%$ in the Middle East [16] to $10-35 \%$ in Europe [17] and up to $50 \%$ in China or Japan [18]. IgAN incidence is probably strongly underestimated, as not every patient with suspected kidney disease undergoes renal biopsy. The striking data from necropsy studies revealed that the prevalence of IgA deposits in the general population may range from $2.4 \%$ to even $16 \%$ [19-21]. The clinical course and outcome of primary IgAN are strongly variable and unpredictable. The symptoms may range from microscopic hematuria (benign condition, usually asymptomatic) to subnephrotic proteinuria to nephrotic proteinuria with gross hematuria (advanced, symptomatic) [22]. Nephrotic range proteinuria, hypertension, decreased estimated glomerular filtration rate (eGFR), and histological grading are robust predictors of adverse renal outcome in
IgA nephropathy [23]. In most cases, the disease progresses over a long period of time and eventually leads to end-stage renal disease (ESRD) in a large proportion of patients (even 50\% within 20 years) [24]. The diagnosis of primary glomerular diseases is challenging and highly complex. It always requires a kidney biopsy, an invasive clinical procedure, associated with a substantial risk (around 3\%) of complications like severe pain, infections, or serious bleeding [25-30]. Additionally, renal biopsy always requires hospital admission and sometimes cannot be performed due to coexisting systemic diseases (e.g., uncontrolled hypertension, atherosclerosis, diabetes mellitus, amyloidosis, and blood coagulation abnormalities) or the lack of patient consent [30]. Other disadvantages include the high cost of the procedure and the time-consuming protocol of sample processing and analysis [31, 32].

Future studies should put great effort into discovering noninvasive, specific biomarkers of primary IgAN that will permit an early detection as well as reliable monitoring and prediction of its course.

\section{Urinary Peptidome}

The peptidome is generally considered to be a fraction of low-molecular weight (LMW) proteome, encompassing amino acid oligomers and polymers, with a molecular mass below $30 \mathrm{kDa}$, that does not require complex processing (e.g., trypsin digestion), prior to mass spectrometry analysis [33-35]. Peptides act as central keepers of homeostasis, affecting and integrating the nervous (neurotransmitters, neuromodulators), endocrine (peptide hormones), and immune system (antimicrobial peptides) [36]. These molecules are mostly generated by the proteolytic breakdown of larger precursor proteins, remaining inactive until cleaved (e.g., the conversion of proinsulin into insulin) [37, 38]. Due to the significantly varying activity of proteases in physiological and pathologic states, the proteolytic peptide pattern may be used to determine the activity of proteases in the context of a specific condition [38]. Recent computational and ribosome profiling studies have revealed that the peptidome also contains numerous peptides, directly translated from short open reading frames (mRNA, lncRNA) [39-41]. The short open reading frame- (sORF-) encoded peptides remain functionally uncharacterised, with the exception of humanin, encoded in the mitochondrial genome (75 bp ORF), and possessing proved neuroprotective and cytoprotective properties [42].

The peptides produced both by normal and diseased body tissues are widely distributed throughout the body fluids, for example, blood, urine, cerebrospinal fluid, and saliva, and comprise an attractive reservoir for biomarker discovery [38]. Urinary proteome/peptidome is of special interest as urine is a noninvasively accessible body fluid that can be obtained repetitively in "large" amounts and most importantly, it is characterised by low proteolytic activity (e.g., in comparison to blood). Urine samples can be stored for hours at room temperature or kept at $4^{\circ} \mathrm{C}$, without significant alterations in the proteomic pattern [43]. This is probably due to the fact that proteolytic degradation has already 
occurred while the urine was "stored" in the bladder [34]. However, if urine is collected for proteomic analysis, particular attention should be paid to the urinary $\mathrm{pH}$, which may significantly contribute to the changes in the urinary peptidomic profile. Previous studies have revealed that a close to neutral urine $\mathrm{pH}$ "assures" protein stability (e.g., due to the activity of trypsin and inter-alpha-trypsin inhibitors), whereas an acidic $\mathrm{pH}(<6.0)$ shifts toward the activation of acid endoproteases (e.g., cathepsin D, aspartic protease) and the generation of "artificial" peptides [12, 44, 45]. The human urine $\mathrm{pH}$ ranges from strongly acidic (4.5) to alkaline (8.0) and is remarkably influenced by diet, medications, and underlying diseases [10]. Thus, urine $\mathrm{pH}$ should be determined immediately after collection and adjusted to neutral, if necessary, to ensure reliable and repeatable results. Apart from a fairly stable peptidomic pattern, another advantage of the urine as a "biomarkers mine" is the "low" complexity and "low" dynamic range of proteins/peptides expression, in comparison to plasma, that is $10^{6}$ for urine and $10^{9}$ for plasma [46, 47]. Normal human urine contains at least 5000 naturally occurring peptides $(<20 \mathrm{kDa})$, originating from glomerular filtration, tubular secretion, and epithelial cells, which line the kidneys and urinary tract $[48,49]$. As much as $70 \%$ of the low-molecular weight proteins come directly from the renal system [50], it is estimated that approximately $49 \%$ of the total urinary proteins/peptides remain associated with the urine supernatant, $48 \%$ belongs to the debris and cell fraction, and around 3\% remains entrapped in the exosomes $[50,51]$. The urinary fraction should be considered as a separate source of potential peptide biomarkers, as they possess diverse protein/peptide composition and provide various types of biological information [51]. The urinary peptidome faithfully reflects the physiological and pathophysiological processes, ongoing in the urinary system, as well as in other parts of the body. Thus, it is widely utilised as a "gold mine" for biomarker discovery for both the urinary system, as well as for nonurinary system-related diseases [7, 52-54].

Urinary peptidomics is a powerful tool, although not free of challenges. Some of the major problems include contamination with organic and inorganic urinary components, persisting in the protein sample even after extensive cleanup and interfering with the mass spectrometry analysis. Another obstacle are the intra- and interindividual differences in protein/peptide concentration requiring complex normalisation strategy. Finally, the exogenous and endogenous variables (e.g., age, smoking, diet, exercise, and environmental factors) significantly alter the proteomic/peptidomic pattern and hamper the urinary biomarker research $[6,55]$.

One of the previous studies showed that coffee consumption is associated with changes in the expression of 11 urinary proteins, including metalloproteinase inhibitor 2 that was earlier considered as a putative biomarker of bladder cancer [56]. Another example of a confounding variable is age. As shown by Zürbig et al., the urinary peptide profile changes significantly with aging; thus, it is critically important to pay attention to the age distribution of discovery and validation cohorts in the course of biomarker discovery projects [48]. In a previous study, by Haubitz et al., the IgAN cohort included $11 \%$ of patients aged above 60 years, thus raising the question of the potential confounding effect of age on the results [6].

Urinary peptidome is certainly an ideal site to search for biomarkers of human diseases, including IgA nephropathy; however, urine proteomics studies have to be planned in detail and critically, by taking into consideration all the potential confounding variables that may influence the accuracy and reliability of the results.

\section{Urinary Peptide Profiles of IgA Nephropathy}

The study published in 2005, by Haubitz et al., provided the first evidence of urinary protein/peptide profile utility in discrimination between IgAN patients, healthy individuals, and membranous nephropathy (MN) subjects and its potential application as a diagnostic tool (Table 1) [6]. The authors also noticed that the IgAN-related profile changes significantly in patients receiving an increasing number of antihypertensive drugs, which implies that urinary peptidome profiling may theoretically serve as a valuable means for evaluating treatment efficacy and testing of novel, promising therapies [6]. The diagnostic potential of the urinary peptidome in IgA nephropathy was further confirmed by Julian et al., who developed a 25-peptide panel, distinctly separating IgAN subjects from healthy controls and from patients with other renal diseases (e.g., FSGS, diabetic nephropathy, and amyloidosis) with an overall specificity of $82.3 \%$ (Table 1) [7]. Unfortunately, the signatures proposed both by Haubitz and Julian have been developed based on relatively small cohorts (specifically IgAN), not properly matched regarding age, using unfractionated urine (Table 1). The analysis of peptides derived from urine supernatant, performed by Graterol et al., resulted in the identification of 16 lowmolecular weight proteins, discriminating between IgAN patients and healthy controls. The signature included uromodulin (UMOD), alpha-1-antitrypsin (A1AT) peptides, and beta-2-microglobulin (B2M) fragment. The level of uromodulin (m/z 1898) and alpha-1-antitrypsin peptide $(\mathrm{m} / \mathrm{z}$ 1945) was found to correlate negatively and positively, respectively, with the lesion severity in IgAN (Table 1). The same group defined a 10-peptide multimarker, composed of UMOD peptides, A1AT fragment, and low-molecular weight proteins, with an unknown amino acid composition that strongly correlated with the doubling of serum creatinine in IgAN patients (Table 1) [57]. However, it should be stressed that the aforementioned study lacked the CKD control group and thus did not evaluate whether the "putative peptide markers" are truly specific for IgA nephropathy. There is currently a high demand for noninvasive biomarkers, possessing the ability to accurately discriminate between different variants of chronic kidney diseases (correlating with histopathological results of renal biopsy), which would enable the monitoring of their clinical course. Another study has revealed that a set of 11 peptides found in urine supernatant possesses diagnostic potential in IgAN. The UMOD (m/z 1913.14) fragment, that was the only wellcharacterised component of the signature, was underrepresented in the urine samples of IgAN subjects in comparison 


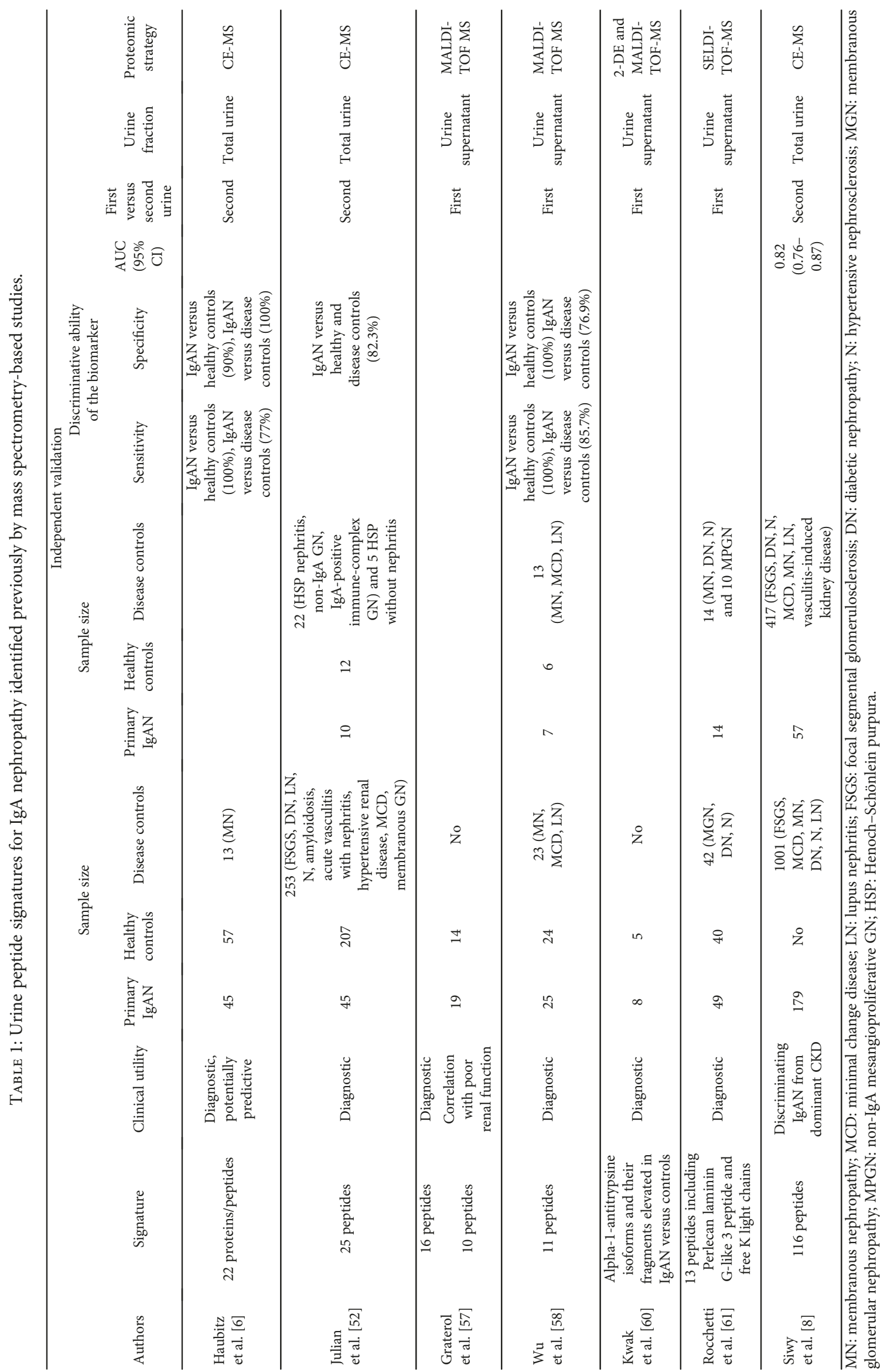


to healthy individuals (Table 1) [58]. Uromodulin is a kidney-specific molecule and the most abundant protein in normal human urine, playing an immunosuppressive role [59]. As the diminished expression of particular UMOD fragments accompanies a number of glomerulopathies, the UMOD level alone cannot be considered as a specific biomarker of IgAN. Another proteomic study found that A1AT protein isoforms and their cleavage products are significantly elevated in urine and renal cortex samples of IgAN patients, in comparison to the healthy control group (Table 1) [60]. A1AT is the main blood serine proteinase inhibitor, possessing a broad spectrum of inhibitory activities and exerting anti-inflammatory effects; thus, its degradation might potentially play a role in IgAN pathogenesis [60]. Unfortunately, the study did not assess the diagnostic value of A1AT, in relation to various types of chronic kidney diseases.

Urinary laminin G-like $3(\mathrm{~m} / \mathrm{z} 21,598)$ and free $\mathrm{K}$ light chains $(\mathrm{m} / \mathrm{z} 23,458)$ are other candidates for peptide biomarkers of IgA nephropathy that were shown to possess the potential to differentiate between IgAN patients, subjects with other chronic kidney diseases, and healthy controls (Table 1). The expression of these peptides was found to correlate inversely with the severity of clinical and histologic features of IgAN, as well as with the clinical outcome [61].

The majority of the previous MS-based peptidomic studies which focused on finding urine peptide biomarkers for IgAN, suffered from insufficient sample sizes and the lack of a truly heterogeneous control group (wide spectrum of renal diseases). The studies conducted so far employed distinct strategies for protein sample preparation and analysis which explains the very small overlap between the "IgAN-specific peptide patterns" originating from distinct studies.

The meta-analysis of proteomic data conducted recently by Siwy et al. represents the first attempt to identify peptide signatures specific for IgAN, by utilising impressively large cohorts. The combined, retrospective analysis of 1180 independent CKD proteomic data sets, yielded a 116-peptide signature, clearly discriminating IgAN condition (179 cases) from other common variants of chronic kidney diseases $(n=1001)$ (Table 1) [8]. The IgAN peptide classifier extracted from merged datasets included a number of protein fragments, recently proposed as potential biomarkers of IgAN [6,7]. 12\% of peptides identified previously by Julian et al. overlapped with the 116 multimarkers, and these were mostly collagen fragments [7]. The diagnostic potential of the proposed signature requires consequent validation in large, multicentre prospective studies that will involve IgAN patients and a broad spectrum of CKD cases.

In a recent study, Good et al. developed a 273 urinary peptide signature (CKD273-classifier), accurately discriminating patients with CKD (independently on aetiology) from healthy and disease controls. The study included an extremely large cohort of 3600 urine samples (including IgAN), collected at several dozen clinical centres in Europe, America, and Australia and processed according to the same standard protocol to assure consistency [43]. This is the first diagnostic peptide signature for CKD, positively validated in prospective studies, which has received a letter of approval from the FDA [62].

\section{Conclusions}

IgA nephropathy is the predominant subtype of primary glomerular disease and one of the most common causes of chronic kidney disease (CKD) worldwide in adults. The current diagnosis of IgAN is based entirely on the evaluation of tissue specimens obtained by kidney biopsy, an invasive diagnostic procedure, carrying a considerable risk of minor and major complications [2, 63]. To date, there are no molecular strategies available that might serve as a noninvasive alternative to the renal biopsy (so-called liquid biopsy). Therefore, there is an urgent need to develop specific and sensitive biomarkers that may be utilised for screening, diagnosis, and prognosis, as well as monitoring of IgAN.

With the advances in mass spectrometry-based proteomics technology in the last decade, it has become possible to globally analyse the protein/peptide composition of various cell types, tissues, and body fluids, for example, blood, serum, urine, amniotic fluid, or cerebrospinal fluid [64]. Urine comprises an excellent biological material for proteomic research, as it can be obtained in large volumes, in a fully noninvasive manner and possesses stable proteome [65]. The urinary peptidome represents the low-molecular weight fraction of the urinary proteome and comprises a valuable source of kidney disease-specific biomarkers, as its pattern changes markedly during the course of the disease $[6,7,9]$.

There are many potential urine peptide biomarkers for IgAN identified to date; however, all of them require further rigorous validation. More studies are mandatory to uncover the qualitative and quantitative profiles of urinary peptides that are related specifically to IgA nephropathy. Future studies should employ large cohorts of IgAN subjects and patients with other chronic kidney diseases, who will represent various and numerous populations. It would be crucial to develop and follow standardised procedures for urine peptidomics (i.e., sample collection, storage, and sample preparation for analysis and quality control), thus ensuring accurate MS-data reproduction by different laboratories. The candidate peptide biomarkers should undergo an extensive interlaboratory validation, to demonstrate their sufficient discriminatory power and suitability for clinical application.

\section{Conflicts of Interest}

The authors declare no competing interests regarding the publication of this paper.

\section{Acknowledgments}

This paper is supported by the Leading National Research Centre (KNOW), for years 2014-2018. 


\section{References}

[1] S. Reid, P. M. Cawthon, J. C. Craig, J. A. Samuels, D. A. Molony, and G. F. Strippoli, "Non-immunosuppressive treatment for IgA nephropathy," Cochrane Database of Systematic Reviews, vol. 16, no. 3, article CD003962, 2011.

[2] M. Fiorentino, D. Bolignano, V. Tesar et al., "Renal biopsy in 2015 - from epidemiology to evidence-based indications," American Journal of Nephrology, vol. 43, no. 1, pp. 1-19, 2016.

[3] L.-P. Laurin, A. Bonnardeaux, M. Dube, and M. Leblanc, "Percutaneous renal biopsy," in Topics in Renal Biopsy and Pathology, M. Mubarak and J. Kazi, Eds., InTech, London, UK, 2012.

[4] J. Bandari, T. W. Fuller, R. M. Turner Ii, and L. A. D'Agostino, "Renal biopsy for medical renal disease: indications and contraindications," The Canadian Journal of Urology, vol. 23, no. 1, pp. 8121-8126, 2016.

[5] X. J. Zhou, Z. Laszik, T. Nadasdy, V. D. D'Agati, and F. G. Silva, Silva's Diagnostic Renal Pathology, CUP, Cambridge, United Kingdom, 2nd edition, 2017.

[6] M. Haubitz, S. Wittke, E. M. Weissinger et al., "Urine protein patterns can serve as diagnostic tools in patients with IgA nephropathy," Kidney International, vol. 67, no. 6, pp. 23132320, 2005.

[7] B. A. Julian, S. Wittke, M. Haubitz et al., "Urinary biomarkers of IgA nephropathy and other IgA-associated renal diseases," World Journal of Urology, vol. 25, no. 5, pp. 467-476, 2007.

[8] J. Siwy, P. Zürbig, A. Argiles et al., "Noninvasive diagnosis of chronic kidney diseases using urinary proteome analysis," Nephrology Dialysis Transplantation, vol. 32, no. 12, pp. 2079-2089, 2016.

[9] C. A. Crutchfield, S. N. Thomas, L. J. Sokoll, and D. W. Chan, "Advances in mass spectrometry-based clinical biomarker discovery," Clinical Proteomics, vol. 13, no. 1, p. 1, 2016.

[10] V. Thongboonkerd, S. Mungdee, and W. Chiangjong, "Should urine $\mathrm{pH}$ be adjusted prior to gel-based proteome analysis?," Journal of Proteome Research, vol. 8, no. 6, pp. 3206-3211, 2009.

[11] T. K. Sigdel, C. D. Nicora, S.-C. Hsieh et al., "Optimization for peptide sample preparation for urine peptidomics," Clinical Proteomics, vol. 11, no. 1, p. 7, 2014.

[12] X. Yang, L. Hu, M. Ye, and H. Zou, "Analysis of the human urine endogenous peptides by nanoparticle extraction and mass spectrometry identification," Analytica Chimica Acta, vol. 829, pp. 40-47, 2014.

[13] S. S. Bellur, S. Troyanov, H. T. Cook, and I. S. Roberts, "Immunostaining findings in IgA nephropathy: correlation with histology and clinical outcome in the Oxford classification patient cohort," Nephrology Dialysis Transplantation, vol. 26, no. 8, pp. 2533-2536, 2011.

[14] Y. Ayar, A. Ersoy, E. Isiktas et al., "The analysis of patients with primary and secondary glomerular diseases: a singlecenter experience," Honk Kong Journal of Nephrology, vol. 19, pp. 28-35, 2016.

[15] A. McGrogan, C. F. Franssen, and C. S. de Vries, "The incidence of primary glomerulonephritis worldwide: a systematic review of the literature," Nephrology, Dialysis, Transplantation, vol. 26, no. 2, pp. 414-430, 2011.

[16] G. Demircin, A. Delibas, K. Bek et al., "A one-center experience with pediatric percutaneous renal biopsy and histopathology in Ankara, turkey," International Urology and Nephrology, vol. 41, no. 4, pp. 933-939, 2009.

[17] I. Kurnatowska, D. Jedrzejka, A. Malyska, M. WagrowskaDanilewicz, M. Danilewicz, and M. Nowicki, "Trends in the incidence of biopsy-proven glomerular diseases in the adult population in central Poland in the years 1990-2010," Kidney \& Blood Pressure Research, vol. 35, no. 4, pp. 254-258, 2012.

[18] H. Sugiyama, H. Yokoyama, H. Sato et al., "Japan renal biopsy registry and japan kidney disease registry: committee report for 2009 and 2010," Clinical and Experimental Nephrology, vol. 17, no. 2, pp. 155-173, 2013.

[19] R. Sinniah, "Occurrence of mesangial IgA and IgM deposits in a control necropsy population," Journal of Clinical Pathology, vol. 36, no. 3, pp. 276-279, 1983.

[20] J. Varis, I. Rantala, and A. Pasternack, "Immunofluorescence of immunoglobulins and complement in kidneys taken at necropsy," Journal of Clinical Pathology, vol. 42, no. 11, pp. 12111214, 1989.

[21] J. Varis, I. Rantala, A. Pasternack et al., "Immunoglobulin and complement deposition in glomeruli of 756 subjects who had committed suicide or met with a violent death," Journal of Clinical Pathology, vol. 46, no. 7, pp. 607-610, 1993.

[22] Y. Caliskan and K. Kiryluk, "Novel biomarkers in glomerular disease," Advances in Chronic Kidney Disease, vol. 21, no. 2, pp. 205-216, 2014.

[23] E. Alamartine, C. Sauron, B. Laurent, A. Sury, A. Seffert, and C. Mariat, "The use of the Oxford classification of IgA nephropathy to predict renal survival," Clinical Journal of the American Society of Nephrology, vol. 6, no. 10, pp. 23842388, 2011.

[24] R. Coppo and G. D'Amico, "Factors predicting progression of IgA nephropathies," Journal of Nephrology, vol. 18, no. 5, pp. 503-512, 2005.

[25] M. Eiro, T. Katoh, and T. Watanabe, "Risk factors for bleeding complications in percutaneous renal biopsy," Clinical and Experimental Nephrology, vol. 9, no. 1, pp. 40-45, 2005.

[26] P. Stratta, C. Canavese, M. Marengo et al., "Risk management of renal biopsy: 1387 cases over 30 years in a single centre," European Journal of Clinical Investigation, vol. 37, no. 12, pp. 954-963, 2007.

[27] K. Toledo, M. J. Pérez, M. Espinosa et al., "Complications associated with percutaneous renal biopsy in Spain, 50 years later," Nefrología, vol. 30, no. 5, pp. 539-543, 2010.

[28] C. Beltrami, A. Clayton, A. O. Phillips, D. J. Fraser, and T. Bowen, "Analysis of urinary microRNAs in chronic kidney disease," Biochemical Society Transactions, vol. 40, no. 4, pp. 875-879, 2012.

[29] M. S. Zhu, J. Z. Chen, and A. P. Xu, "Factors that can minimize bleeding complications after renal biopsy," International Urology and Nephrology, vol. 46, no. 10, pp. 1969-1975, 2014.

[30] A. Sumnu, M. Gursu, and S. Ozturk, "Primary glomerular diseases in the elderly," World Journal of Nephrology, vol. 4, no. 2, pp. 263-270, 2015.

[31] S. Hu, J. A. Loo, and D. T. Wong, "Human body fluid proteome analysis," Proteomics, vol. 6, no. 23, pp. 6326-6353, 2006.

[32] V. Romanov, T. C. Whyard, W. C. Waltzer, and F. S. Darras, "A noninvasive assay for monitoring renal allograft status," Journal of Biological Methods, vol. 1, no. 1, article e2, 2014.

[33] R. R. Drake, L. Cazares, and O. J. Semmes, "Mining the low molecular weight proteome of blood," Proteomics. Clinical Applications, vol. 1, no. 8, pp. 758-768, 2007. 
[34] H. Mischak, B. A. Julian, and J. Novak, "High-resolution proteome/peptidome analysis of peptides and low-molecularweight proteins in urine," Proteomics. Clinical Applications, vol. 1, no. 8, pp. 792-804, 2007.

[35] S. Decramer, A. Gonzalez de Peredo, B. Breuil et al., "Urine in clinical proteomics," Molecular \& Cellular Proteomics, vol. 7, no. 10, pp. 1850-1862, 2008.

[36] A. Mor, Peptides: Biological Activities of Small Peptides, eLS, Indianapolis, IN, USA, 2001.

[37] A. Varro, Posttranslational Processing: Peptide Hormones and Neuropeptide Transmitters, eLS, Indianapolis, IN, USA, 2007.

[38] J. M. Bauca, E. Martínez-Morillo, and E. P. Diamandis, "Peptidomics of urine and other biofluids for cancer diagnostics," Clinical Chemistry, vol. 60, no. 8, pp. 1052-1061, 2014.

[39] S. A. Slavoff, A. J. Mitchell, A. G. Schwaid et al., "Peptidomic discovery of short open reading frame-encoded peptides in human cells," Nature Chemical Biology, vol. 9, no. 1, pp. 5964, 2013.

[40] J. Ruiz-Orera, X. Messeguer, J. A. Subirana, and M. M. Alba, "Long non-coding RNAs as a source of new peptides," eLife, vol. 3, no. 3, article e03523, 2014.

[41] D. M. Anderson, K. M. Anderson, C. L. Chang et al., "A micropeptide encoded by a putative long noncoding RNA regulates muscle performance," Cell, vol. 160, no. 4, pp. 595606, 2015.

[42] Y. Hashimoto, Y. Ito, T. Niikura et al., "Mechanisms of neuroprotection by a novel rescue factor humanin from Swedish mutant amyloid precursor protein," Biochemical and Biophysical Research Communications, vol. 283, no. 2, pp. 460-468, 2001.

[43] D. M. Good, P. Zürbig, A. Argilés et al., "Naturally occurring human urinary peptides for use in diagnosis of chronic kidney disease," Molecular \& Cellular Proteomics, vol. 9, no. 11, pp. 2424-2437, 2010.

[44] S. Schaub, J. A. Wilkins, M. Antonovici et al., "Proteomic-based identification of cleaved urinary $\beta 2$-microglobulin as a potential marker for acute tubular injury in renal allografts," American Journal of Transplantation, vol. 5, no. 4, pp. 729-738, 2005.

[45] C. Rose, A. Parker, B. Jefferson, and E. Cartmell, "The characterization of feces and urine: a review of the literature to inform advanced treatment technology," Critical Reviews in Environmental Science and Technology, vol. 45, no. 17, pp. 1827-1879, 2015.

[46] R. Pieper, C. L. Gatlin, A. J. Makusky et al., “The human serum proteome: display of nearly 3700 chromatographically separated protein spots on two-dimensional electrophoresis gels and identification of 325 distinct proteins," Proteomics, vol. 3, no. 7, pp. 1345-1364, 2003.

[47] N. Nagaraj and M. Mann, "Quantitative analysis of the intra- and inter-individual variability of the normal urinary proteome," Journal of Proteome Research, vol. 10, no. 2, pp. 637-645, 2011.

[48] P. Zürbig, S. Decramer, M. Dakna et al., "The human urinary proteome reveals high similarity between kidney aging and chronic kidney disease," Proteomics, vol. 9, no. 8, pp. 21082117, 2009.

[49] C. Wiesner, C. Hannum, K. Reckamp et al., "Consistency of a two clinical site sample collection: a proteomics study," Proteomics. Clinical Applications, vol. 4, no. 8-9, pp. 726738, 2010.
[50] S. Kalantari, A. Jafari, R. Moradpoor, E. Ghasemi, and E. Khalkhal, "Human urine proteomics: analytical techniques and clinical applications in renal diseases," International Journal of Proteomics, vol. 2015, Article ID 782798, 17 pages, 2015.

[51] H. Zhou, P. S. Yuen, T. Pisitkun et al., "Collection, storage, preservation, and normalization of human urinary exosomes for biomarker discovery," Kidney International, vol. 69, no. 8, pp. 1471-1476, 2006.

[52] B. A. Julian, S. Wittke, J. Novak et al., "Electrophoretic methods for analysis of urinary polypeptides in IgAassociated renal diseases," Electrophoresis, vol. 28, no. 23, pp. 4469-4483, 2007.

[53] D. Theodorescu, S. Wittke, M. M. Ross et al., "Discovery and validation of new protein biomarkers for urothelial cancer: a prospective analysis," The Lancet Oncology, vol. 7, no. 3, pp. 230-240, 2006.

[54] E. R. Smith, D. Zurakowski, A. Saad, R. M. Scott, and M. A. Moses, "Urinary biomarkers predict brain tumor presence and response to therapy," Clinical Cancer Research, vol. 14, no. 8, pp. 2378-2386, 2008.

[55] E. Schiffer, H. Mischak, and J. Novak, "High resolution proteome/peptidome analysis of body fluids by capillary electrophoresis coupled with MS," Proteomics, vol. 6, no. 20, pp. 5615-5627, 2006.

[56] Y. Ni, F. Zhang, M. An, W. Yin, and Y. Gao, "Acute effect of coffee consumption on urinary proteome in young healthy individuals," MOJ Proteomics \& Bioinformatics, vol. 5, no. 2, article 00153, 2017.

[57] F. Graterol, M. Navarro-Muñoz, M. Ibernon et al., "Poor histological lesions in IgA nephropathy may be reflected in blood and urine peptide profiling," BMC Nephrology, vol. 14, no. 1, p. 82, 2013.

[58] J. Wu, N. Wang, J. Wang et al., "Identification of a uromodulin fragment for diagnosis of IgA nephropathy," Rapid Communications in Mass Spectrometry, vol. 24, no. 14, pp. 1971-1978, 2010.

[59] C. Hession, J. M. Decker, A. P. Sherblom et al., "Uromodulin (Tamm-Horsfall glycoprotein): a renal ligand for lymphokines," Science, vol. 237, no. 4821, pp. 1479-1484, 1987.

[60] N. J. Kwak, E.-H. Wang, I. Y. Heo et al., "Proteomic analysis of alpha-1-antitrypsin in immunoglobulin a nephropathy," Proteomics. Clinical Applications, vol. 1, no. 4, pp. 420-428, 2007.

[61] M. T. Rocchetti, M. Papale, A. M. d'Apollo et al., "Association of urinary laminin G-like 3 and free $\mathrm{K}$ light chains with disease activity and histological injury in IgA nephropathy," Clinical Journal of the American Society of Nephrology, vol. 8, no. 7, pp. 1115-1125, 2013.

[62] http://www.fda.gov/downloads/Drugs/DevelopmentApproval Process/UCM508790.pdf.

[63] L. González-Michaca, A. Chew-Wong, L. Soltero, G. Gamba, and R. Correa-Rotter, "Percutaneous kidney biopsy, analysis of 26 years: complication rate and risk factors; comment," Revista de Investigación Clínica, vol. 52, no. 2, pp. 125-131, 2000.

[64] M. Wilhelm, J. Schlegl, H. Hahne et al., "Mass-spectrometrybased draft of the human proteome," Nature, vol. 509, no. 7502, pp. 582-587, 2014.

[65] G. Schley, C. Köberle, E. Manuilova et al., "Comparison of plasma and urine biomarker performance in acute kidney injury," PLoS One, vol. 10, no. 12, article e0145042, 2015. 


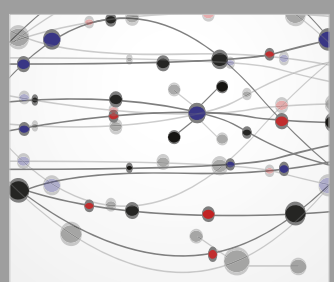

The Scientific World Journal
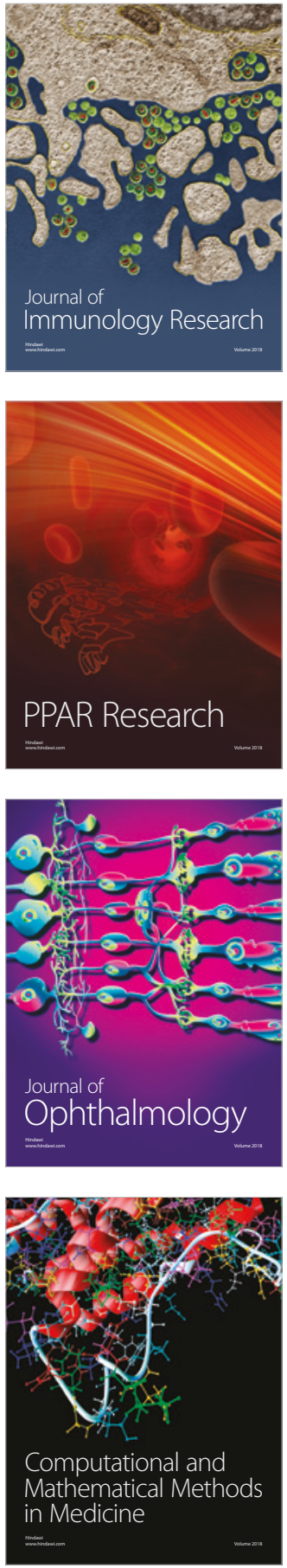

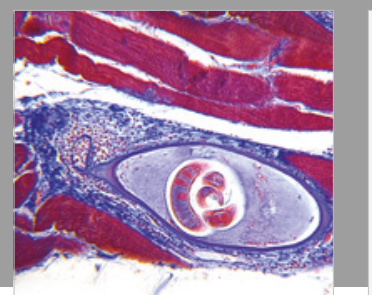

Gastroenterology Research and Practice

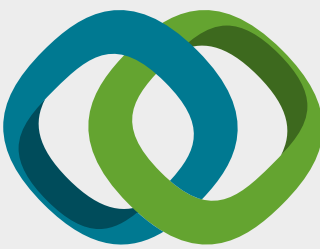

\section{Hindawi}

Submit your manuscripts at

www.hindawi.com
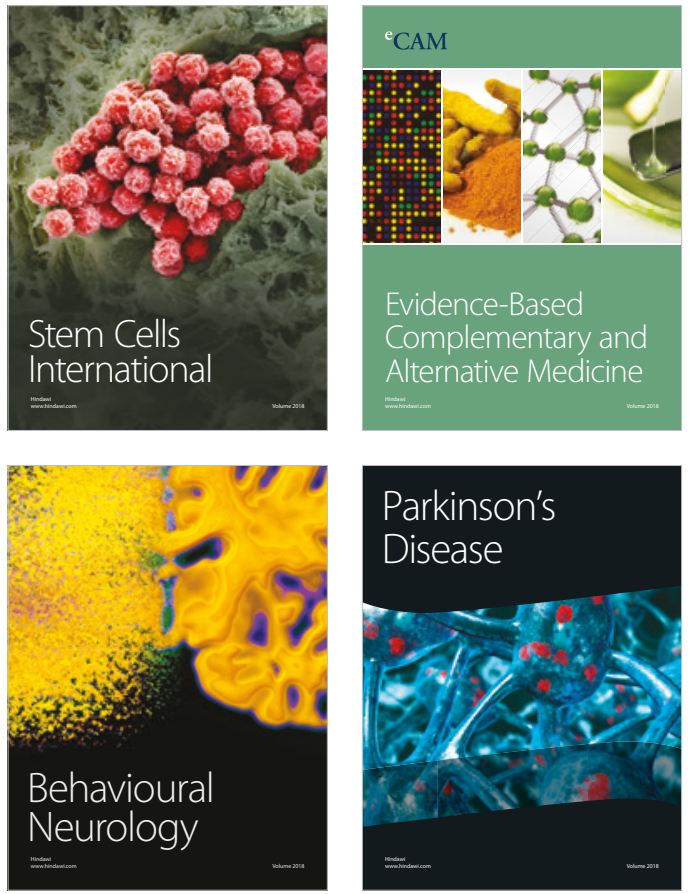

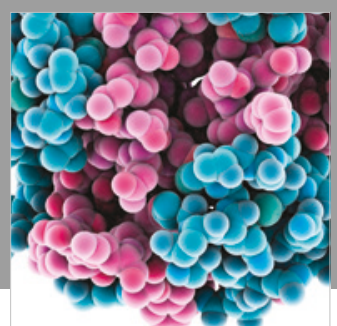

ournal of

Diabetes Research

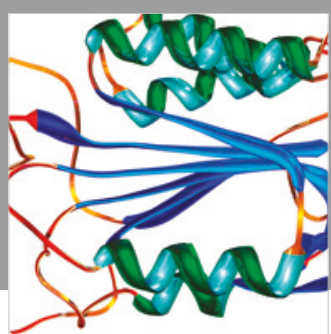

Disease Markers
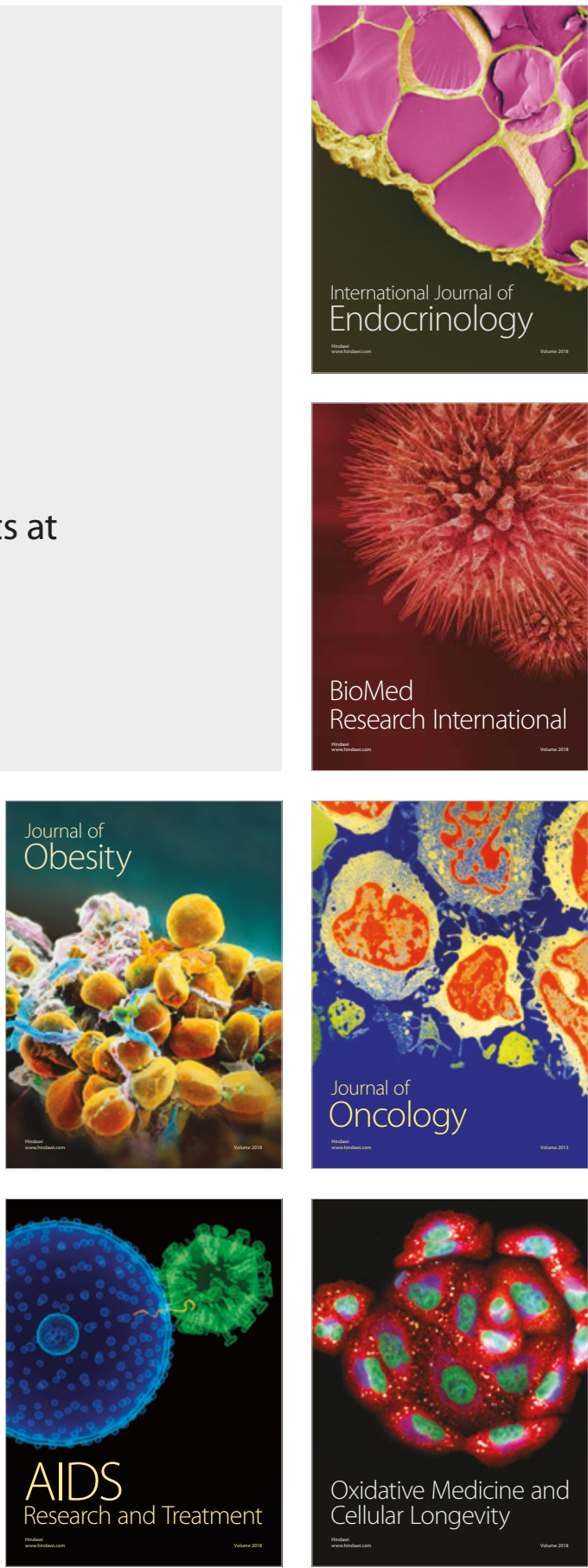